\title{
The Casimir effect for parallel plates in the spacetime with a fractal extra compactified dimension
}

\author{
Hongbo Cheng* \\ Department of Physics, East China University of Science and Technology, \\ Shanghai 200237, China \\ The Shanghai Key Laboratory of Astrophysics, \\ Shanghai 200234, China
}

\begin{abstract}
The Casimir effect for massless scalar fields satisfying Dirichlet boundary conditions on the parallel plates in the presence of one fractal extra compactified dimension is analyzed. We obtain the Casimir energy density by means of the regularization of multiple zeta function with one arbitrary exponent. We find a limit on the scale dimension like $\delta>\frac{1}{2}$ to keep the negative sign of the renormalized Casimir energy which is the difference between the regularized energy for two parallel plates and the one with no plates. We derive and calculate the Casimir force relating to the influence from the fractal additional compactified dimension between the parallel plates. The larger scale dimension leads to the greater revision on the original Casimir force. The two kinds of curves of Casimir force in the case of integer-numbered extra compactified dimension or fractal one are not superposition, which means that the Casimir force show whether the dimensionality of additional compactified space is integer or fraction.
\end{abstract}

PACS number(s): 04.50.+h, 11.10Kk, 04.62.+v

*E-mail address: hbcheng@sh163.net 
During the investigation of quantum gravity, more attentions have been paid to the fractal universe [1]. It may be better to describe the spacetime whose scale is on the Planck order in virtue of fractal geometry with some non-integer dimensions. Within this kind of background some topics were considered. It is demonstrated that the spectral dimension of the spacetime where Quantum Einstein Gravity lives in is equal to 2 microscopically while to 4 on macroscopic scales [2]. It is also shown that the world with a quantum group symmetry has a scale-dependent fractal dimension at short scales to describe a phenomenon appeared in the quantum gravity [3]. A kind of field theory which is Lorentz invariant, power-counting renormalizable, ultraviolet finite and causal is proposed to search for a consistent theory of quantum gravity $[4,5]$.

More than 80 years ago Kaluza and Klein put forward the model that our universe has more than four dimensions $[6,7]$. The extra spatial dimensions belonging to the modern Kaluza-Klein theory are chosen to be compact and small to unify all interactions in nature. Their characteristic size is of the order of the Planck length. In addition the quantum gravity such as string theory or brane-world scenario is developed to reconcile the quantum mechanics and gravity with the help of introducing the additional spatial dimensions. It seems to be reasonable to describe the extra space by means of fractal geometry because of the quantum fluctuations on the Planck scale space. The Kaluza-Klein model with non-integer number of additional dimensions needs to be developed in particular in various directions. The Kaluza-Klein theory involving a fractal extra dimension $D$ within the range $0<D<2$ was put forward in Ref. [8].

A resemble macroscopic quantum effect describing the attractive force between two conducting and neutral parallel plates was put forward by Casimir more than 60 years ago [9-12]. The Casimir effect appears due to the disturbance of the vacuum of the electromagnetic field induced by the presence of boundary. The Casimir effect becomes a powerful tool to research on the model of Universe with more than four dimensions because the effect has something to do with the additional dimensions and the precision of the effect measurement has been greatly improved practically [13-16] and the effect is an observable and trustworthy consequence of the existence of quantum fluctuations. More efforts have been made to the Casimir effect for parallel plates within the frame of Kaluza-Klein model with the integer number of additional dimensions [17-29]. It has been shown that the extra-dimension influence was manifest and distinct. It is significant and practical to study the Casimir effect for parallel plates in the spacetimes involving fractal extra compactified dimensions. We wonder how the influence from fractal extra dimensions on the Casimir effect for the parallel-plate system. This problem, to our knowledge, has not been considered. As a starting point and for simplicity, we choose that the background has one fractal additional compactified dimension. The main purpose of this letter is to explore the Casimir effect for two parallel plates in the spacetime with one fractal extra compactified spatial dimension. We obtain the expressions of the total vacuum energy and force respectively. Further we obtain the Casimir energy and Casimir force by means of the zeta-function regularization $[30,31]$ that is used to leave finite results via throwing the divergent terms and our results are not regularization dependent. During the process 
we will perform the regularization of multiple zeta function with one arbitrary exponent again. We will focus on the influence from fractal dimensionality of the additional space on the Casimir effect for two parallel plates. The fractal dimensionality is within the limit between 0 and 2 instead of being exact like an integer. We hope to constrain that. Our discussions and conclusions are listed in the end.

The content of the fractal extra compactified dimension in the Kaluza-Klein theory were introduced in Ref. [8]. According to fractal geometry the scale dimension $\delta=D-D_{T}$ is defined as,

$$
\delta \equiv \frac{d(\ln L)}{d\left(\ln \frac{l}{\lambda}\right)}
$$

where $L$ is the main fractal variable denoting a length of a fractal curve, an area of a fractal surface, etc.. The coefficient $l$ is the measurement scale. The coefficient $\lambda$ is the resolution of the measurement. Here $D$ is the fractal dimension. $D_{T}$ is the topological dimension and $D_{T}=1$ for a curve, $D_{T}=2$ for a surface. According to Eq. (1) that $\delta$ is a constant leads,

$$
L=L_{0}\left(\frac{l}{\lambda}\right)^{\delta}
$$

where the length $L_{0}$ is measured when $\lambda=l$ and of the order of the Planck length. Within the Kaluza-Klein issue the field must be periodic in the fifth coordinate denoted as $x^{5}$, leading to the appearance of an infinite tower of solutions with a quantized $x^{5}$ component of the momentum like $q_{n}=\frac{2 \pi n}{L}$ where $L$ satisfies Eq. (2). It may be assumed that the measurement scale $\lambda$ in Eq. (2) corresponds to $\frac{2 \pi}{q_{n}}$ since any constant factor may be absorbed in the value of $l$. The equation is obtained as follow,

$$
q_{n} L_{0}\left(\frac{l q_{n}}{2 \pi}\right)^{\delta}=2 \pi n
$$

Certainly the spectrum of momentum is

$$
q_{n}=2 \pi\left(\frac{n}{L_{0} l^{\delta}}\right)^{\frac{1}{1+\delta}}
$$

where $n$ is a nonnegative integer. This tower of solutions will recover to be the tower in the regular five-dimensional Kaluza-Klein theory if we choose $\delta=0$.

We start to consider the massless scalar field in the two-parallel-plate system in the spacetime with a non-integer numbered extra compactified dimension. The field obeys the Dirichlet condition, leading the wave vector in the directions restricted by the plates to be $k_{n}=\frac{n \pi}{R}, n$ a positive integer and $R$ the separation of the two plates. In the case of a fractal additional compactified dimension we find the frequency of the vacuum fluctuation within a region confined by two parallel plates whose separation is $R$ to be,

$$
\omega_{N n}=\sqrt{k^{2}+\frac{N^{2} \pi^{2}}{R^{2}}+\frac{(2 \pi)^{2}}{\left(L_{0} l^{\delta}\right)^{\frac{2}{1+\delta}}} n^{\frac{2}{1+\delta}}}
$$


where

$$
k^{2}=k_{1}^{2}+k_{2}^{2}
$$

Here we employ the momentum of the fifth coordinate under the fractal geometry presented in Eq. (4). $k_{1}$ and $k_{2}$ are wave vectors in directions of the unbound space coordinates parallel to the plates surface. Now $n$ is a nonnegative integer. According to Ref. [10-12, 30,31] the total energy density of the fields in the interior of two-parallel-plate device reads,

$$
\begin{aligned}
& \varepsilon\left(R, L_{0}, \delta\right)=\int \frac{d^{2} k}{(2 \pi)^{2}} \sum_{N=1}^{\infty} \sum_{n=0}^{\infty} \frac{1}{2} \omega_{N n} \\
= & \frac{\pi^{2}}{4 R^{3}} \frac{\Gamma\left(-\frac{3}{2}\right) \zeta(-3)}{\Gamma\left(-\frac{1}{2}\right)}+\frac{1}{4 \pi} \frac{\Gamma\left(-\frac{3}{2}\right)}{\Gamma\left(-\frac{1}{2}\right)} M_{2}\left(-\frac{3}{2} ; \frac{\pi^{2}}{R^{2}}, \frac{(2 \pi)^{2}}{\left(L_{0} l^{\delta}\right)^{\frac{2}{1+\delta}}} ; 2, \frac{2}{1+\delta}\right)
\end{aligned}
$$

in terms of the multiple zeta function with one arbitrary exponent. The general multiple zeta function with arbitrary exponents $M_{2}\left(s ; a_{1}, a_{2} ; \alpha_{1}, \alpha_{2}\right)$ is defined as,

$$
M_{2}\left(s ; a_{1}, a_{2} ; \alpha_{1}, \alpha_{2}\right)=\sum_{n_{1}, n_{2}=1}^{\infty}\left(a_{1} n_{1}^{\alpha_{1}}+a_{2} n_{2}^{\alpha_{2}}\right)^{-s}
$$

This kind of zeta function has been regularized with neglecting the small contribution in Ref. [30, 31]. Here we utilize the standard method to regularize the multiple zeta function with one arbitrary exponent $M_{2}\left(s ; a_{1}, a_{2} ; 2, \alpha_{2}\right)$ which will be used in this work as follow,

$$
\begin{aligned}
& M_{2}\left(s ; a_{1}, a_{2} ; 2, \alpha_{2}\right) \\
= & \sum_{n_{1}, n_{2}=1}^{\infty}\left(a_{1} n_{1}^{2}+a_{2} n_{2}^{\alpha_{2}}\right)^{-s} \\
= & -\frac{1}{2} a_{2}^{-s} \zeta\left(\alpha_{2} s\right)+\frac{1}{2} a_{2}^{-s} \sqrt{\frac{\pi a_{2}}{a_{1}}} \frac{\Gamma\left(s-\frac{1}{2}\right)}{\Gamma(s)} \zeta\left(\alpha_{2}\left(s-\frac{1}{2}\right)\right) \\
& +2 \pi^{s} a_{1}^{-\frac{s}{2}-\frac{1}{4}} a_{2}^{-\frac{s}{2}+\frac{1}{4}} \sum_{n_{1}, n_{2}=1}^{\infty}\left(\frac{n_{1}}{n_{2}^{\frac{\alpha_{2}}{2}}}\right)^{s-\frac{1}{2}} K_{-\left(s-\frac{1}{2}\right)}\left(2 \pi \sqrt{\frac{a_{2}}{a_{1}}} n_{1} n_{2}^{\frac{\alpha_{2}}{2}}\right)
\end{aligned}
$$

where $K_{\nu}(z)$ is the modified Bessel function of the second kind and drops exponentially with $z$. We keep the small contribution denoted as the modified Bessel function term. We make use of the regularization of the multiple zeta function with one arbitrary exponent in Eq. (9) to obtain the Casimir energy per unit area of a system consisting of two parallel plates in the spacetime with one fractal extra compactified spatial dimension,

$$
\begin{gathered}
\varepsilon_{C}=-\frac{\pi^{2}}{720} \frac{1}{R^{3}}+\frac{1}{2 \pi^{\frac{3}{2}}} \frac{1}{\left(L_{0} l^{\delta}\right)^{\frac{3}{1+\delta}}} \Gamma\left(-\frac{3}{2}\right) \zeta\left(-\frac{3}{1+\delta}\right)-\pi^{2} \frac{R}{\left(L_{0} l^{\delta}\right)^{\frac{4}{1+\delta}}} \Gamma(-2) \zeta\left(-\frac{4}{1+\delta}\right) \\
-\frac{1}{R\left(L_{0} l^{\delta}\right)^{\frac{2}{1+\delta}}} \sum_{n_{1}, n_{2}=1}^{\infty}\left(\frac{n_{2}^{\frac{1}{1+\delta}}}{n_{1}}\right)^{2} K_{2}\left(4 \pi \frac{R}{\left(L_{0} l^{\delta}\right)^{\frac{1}{1+\delta}}} n_{1} n_{2}^{\frac{1}{1+\delta}}\right)
\end{gathered}
$$


It is clear that the $\Gamma(-2)$ term is equal to infinity. According to the procedure in Ref. [32] we can subtract the part without plates to renormalize the Casimir energy density because the subtracted part compensates exactly the divergent term involving $\Gamma(-2)$. In the absence of plates the vacuum energy density is $\varepsilon_{0}=\int \frac{d^{3} \kappa}{(2 \pi)^{3}} \sum_{n=0}^{\infty} \frac{1}{2} \sqrt{\kappa^{2}+\frac{(2 \pi)^{2}}{\left(L_{0} l^{\delta}\right)^{\frac{2}{1+\delta}}} n^{\frac{2}{1+\delta}}}=\pi^{2} \Gamma(-2) \zeta\left(-\frac{4}{1+\delta}\right) \frac{1}{\left(L_{0} l^{\delta}\right)^{\frac{4}{(1+\delta)}}}$. The expression for the renormalized Casimir energy density becomes,

$$
\begin{gathered}
\varepsilon_{C}^{r e n}=\varepsilon_{C}-R \varepsilon_{0} \\
=-\frac{\pi^{2}}{720} \frac{1}{R^{3}}-\frac{\pi^{\frac{\delta-2}{1+\delta}}}{2(1+\delta)} \frac{\Gamma\left(\frac{3}{2(1+\delta)}\right) \Gamma\left(\frac{4+\delta}{2(1+\delta)}\right)}{\Gamma\left(\frac{3}{2}\right)} \zeta\left(\frac{4+\delta}{1+\delta}\right)\left(\sin \frac{3 \pi}{2(1+\delta)}\right) \frac{1}{\left(L_{0} l^{\delta}\right)^{\frac{3}{1+\delta}}} \\
-\frac{1}{R\left(L_{0} l^{\delta}\right)^{\frac{2}{1+\delta}}} \sum_{n_{1}, n_{2}=1}^{\infty}\left(\frac{n_{2}^{\frac{1}{1+\delta}}}{n_{1}}\right)^{2} K_{2}\left(4 \pi \frac{R}{\left(L_{0} l^{\delta}\right)} n_{1} n_{2}^{\frac{1}{1+\delta}}\right)
\end{gathered}
$$

If the plate distance is extremely large, the renormalized Casimir energy density reduces to be

$$
\lim _{R \longrightarrow \infty} \varepsilon_{C}^{r e n}=-\frac{\pi^{\frac{\delta-2}{1+\delta}}}{2(1+\delta)} \frac{\Gamma\left(\frac{3}{2(1+\delta)}\right) \Gamma\left(\frac{4+\delta}{2(1+\delta)}\right)}{\Gamma\left(\frac{3}{2}\right)} \zeta\left(\frac{4+\delta}{1+\delta}\right)\left(\sin \frac{3 \pi}{2(1+\delta)}\right) \frac{1}{\left(L_{0} l^{\delta}\right)^{\frac{3}{1+\delta}}}
$$

In order to keep the negative nature of the Casimir energy, it is necessary to choose that $\sin \frac{3 \pi}{2(1+\delta)}>$ 0 corresponding to

$$
\delta>\frac{1}{2}
$$

The number of the fractal additional dimension varies between 0 and 2 , or equivalently the scale dimension is within the range $-1<\delta<1$. Now we give rise to a constrain on the parameter to narrow the region like $\delta \in\left(\frac{1}{2}, 1\right)$.

By means of the differentiation rule for the modified Bessel functions $\frac{\partial}{\partial z} K_{\nu}(z)=-\frac{1}{2}\left[K_{\nu-1}(z)+\right.$ $\left.K_{\nu+1}(z)\right]$ we take the derivative of the renormalized Casimir energy density with respect to the plates distance to obtain the Casimir force per unit plate area between them as,

$$
\begin{aligned}
& f_{C}=-\frac{\partial \varepsilon_{C}^{r e n}}{\partial R} \\
& =-\frac{\pi^{2}}{240} \frac{1}{\mu^{4}} \frac{1}{\left(L_{0} l^{\delta}\right)^{\frac{4}{1+\delta}}}+C(\mu, \delta)
\end{aligned}
$$

where the correction function $C(\mu, \delta)$ is expressed as,

$$
\begin{aligned}
C(\mu, \delta)=- & \frac{1}{\mu^{2}} \sum_{n_{1}, n_{2}=1}^{\infty}\left(\frac{n_{2}^{\frac{1}{1+\delta}}}{n_{1}}\right)^{2} K_{2}\left(4 \pi \mu n_{1} n_{2}^{\frac{1}{1+\delta}}\right) \\
& \left.-\frac{2 \pi}{\mu} \sum_{n_{1}, n_{2}=1}^{\infty} \frac{n_{2}^{\frac{3}{1+\delta}}}{n_{1}}\left[K_{1}\left(4 \pi \mu n_{1} n_{2}^{\frac{1}{1+\delta}}\right)+K_{3}\left(4 \pi \mu n_{1} n_{2}^{\frac{1}{1+\delta}}\right)\right]\right\} \frac{1}{\left(L_{0} l^{\delta}\right)^{\frac{4}{1+\delta}}}
\end{aligned}
$$


and

$$
\mu=\frac{R}{\left(L_{0} l^{\delta}\right)^{\frac{1}{1+\delta}}}
$$

It should be emphasized that the first term named as $f_{0}=-\frac{\pi^{2}}{240} \frac{1}{\mu^{4}} \frac{1}{\left(L_{0} l^{\delta}\right)^{\frac{4}{1+\delta}}}$ in Eq. (14) is the same as Casimir pressure on the parallel plates involving massless scalar fields satisfying the Dirichlet conditions in the four-dimensional spacetimes. It should be pointed out that the correction function represents the deviation from the fractal additional dimension. When the plates gap is larger enough than the size of the extra dimension, the correction function approaches to the zero,

$$
\lim _{\mu \longrightarrow \infty} C(\mu, \delta)=0
$$

no matter what the value of $\delta$ is equal to. For a definite value of $\delta$ the behaviour of the correction function on the variable $\mu$ is plotted in Fig. 1. The shapes of the function for various values of scale dimension $\delta$ within the range as Eq. (13) respectively are similar, but they are not superposition. In order to show how great the influence of fractal extra dimension modifies the original Casimir force between the parallel plates we introduce an ratio as a function of $\mu$ and $\delta$,

$$
q(\mu, \delta)=\frac{C(\mu, \delta)}{f_{0}}
$$

We show the function $q(\mu, \delta)$ for a definite value of $\delta$ in Fig. 2. It is manifest that the ratio $q(\mu, \delta)$ is a decreasing function of $\mu$. In Fig. 3 we demonstrate the curves of the ratio as the functions of $\mu$ for some different values of scale dimension $\delta$. We discover that the value of the ratio becomes greater wholly when the scale dimension is larger, which means that the greater scale dimension will bring about stronger influence from the fractal additional dimension. Having compared our recent results with those in Ref. [23-25] we find that the magnitude of the Casimir force in the case of integer number of additional dimension and in the fractal one are different. Within the scope that measurement reaches the enough precision, we can distinguish whether the revisions are from the extra compactified space with integer dimensionality or fractal one.

In this letter the Casimir effect for parallel plates in the spacetime with one fractal extra compactified dimension is discussed. We obtain the total vacuum energy density according to the Kaluza-Klein with one non-integer dimension [8]. We perform the regularization of multiple zeta function with one arbitrary exponent in order to regularize the total vacuum energy density to obtain the Casimir energy density. It is found that the sign of the renormalized Casimir energy remains negative if the scale dimension $\delta>\frac{1}{2}$. The renormalized Casimir energy density is the difference between the Casimir energy density within two parallel plates and the one in the absence of plates. The expression of Casimir force per unit area on the plates is also obtained. Certainly the Casimir force has something to do with the extra spatial dimensions. The larger scale dimension leads the greater modification on the Casimir force. As the separation of the two parallel plates is larger enough than the size of fractal extra dimension, the fractal-extra-dimension influence on the 
Casimir force will be weaker. The curves of the Casimir force for parallel plates in the presence of additional compactified space with integer-numbered dimension or fractal-numbered dimension are not superposition. They are distinct. We open up a new direction to detect whether the dimensionality of extra compactified space is integer or fractal if the experimental device reaches to be sufficiently sensitive. Our research can be generalized to the case that the background has multiple fractal additional compactified spatial dimension.

\section{Acknowledgement}

This work is supported by NSFC No. 10875043 and is partly supported by the Shanghai Research Foundation No. 07dz22020. 


\section{References}

[1] J. Ambjorn, J. Jurkiewicz, R. Loll, Phys. Rev. D72(2005)064014

[2] O. Lauscher, M. Reuter, JHEP0510(2005)050

[3] D. Benedetti, Phys. Rev. Lett. 102(2009)111303

[4] G. Calcagni, Phys. Rev. Lett. 104(2010)251301

[5] G. Calcagni, Phys. Lett. B697(2011)251

[6] T. Kaluza, Sitz. Preuss. Akad. Wiss. Phys. Math. K1. 1(1921)966

[7] O. Klein, Z. Phys. 37(1926)895

[8] I. I. Smolyaninov, Phys. Rev. D65(2002)047503

[9] H. B. G. Casimir, Proc. K. Ned. Akad. Wet. 51(1948)793

[10] M. Bordag, U. Mohideen, V. M. Mostepanenko, Phys. Rep. 353(2001)1

[11] K. A. Milton, The Casimir Effect, Physical Manifestation of Zero-Point Energy, World Scientific, Singapore, 2001

[12] V. M. Mostepanenko, N. N. Trunov, The Casimir Effect and its Applications, Oxford University Press, Oxford, 1997

[13] S. K. Lamoreaux, Phys. Rev. Lett. 78(1997)5

[14] U. Mohideen, A. Roy, Phys. Rev. Lett. 81(1998)4549

[15] G. Bressi, G. Carugno, R. Onfrio, G. Ruoso, Phys. Rev. Lett. 88(2002)041804

[16] R. S. Decca, D. Lepez, E. Fischbach, D. E. Kraus, Phys. Rev. Lett. 91(2003)050402

[17] K. Poppenhaeger, S. Hossenfelder, S. Hofmann, M. Bleicher, Phys. Lett. B582(2004)1

[18] H. Cheng, Mod. Phys. Lett. A21(2006)1957

[19] H. Cheng, Phys. Lett. B643(2006)311

[20] M. P. Hertzberg, R. L. Jaffe, M. Kardar, A. Scardicchio, Phys. Rev. Lett. 95(2005)250402

[21] A. Edery, Phys. Rev. D75(2007)105012

[22] A. Edery, V. Marachevsky, JHEP0812(2008)035

[23] H. Cheng, Phys. Lett. B668(2008)72

[24] K. Kirsten, S. A. Fulling, Phys. Lett. B671(2009)179 
[25] K. Kirsten, S. A. Fulling, Phys. Rev. D79(2009)065019

[26] K. Milton, J. Wagner, Phys. Rev. D80(2009)125028

[27] E. Elizalde, S. D. Odintsov, A. A. Saharian, Phys. Rev. D79(2009)065023

[28] L. P. Teo, Phys. Lett. B682(2009)259

[29] M. Rypestol, I. Brevik, New J. Phys. 12(2010)013022

[30] E. Elizalde, S. D. Odintsov, A. Romeo, A. A. Bysenko, S. Zerbini, Zeta Regularization Techniques with Applications, World Scientific, Singapore, 1994

[31] E. Elizalde, Ten Physical Applications of Spectral Zeta Functions, Springer-Verlag, Berlin, 1995

[32] M. Frank, I. Turan, L. Ziegler, Phys. Rev. D76(2007)015008 


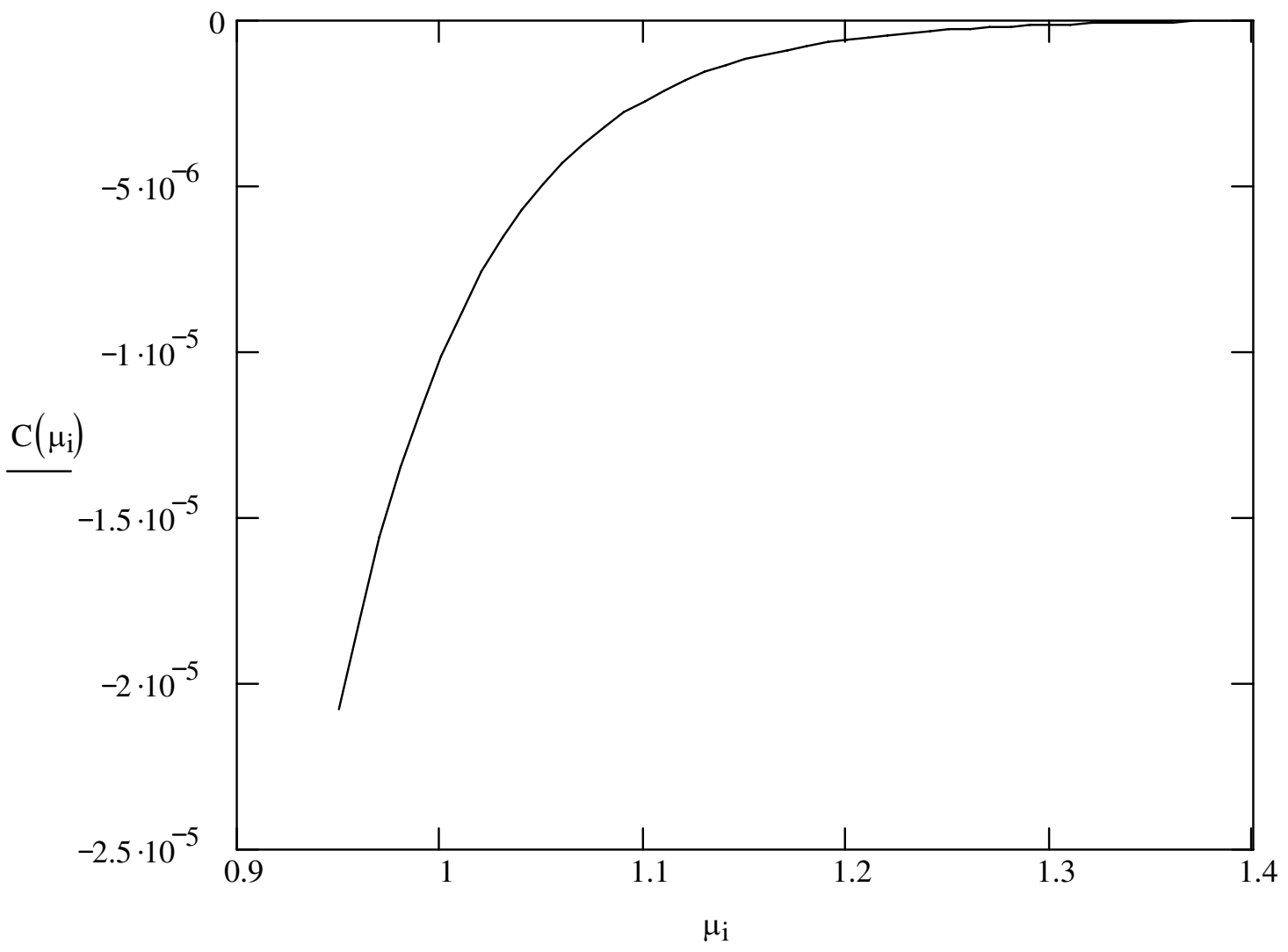

Figure 1: The curve of the correction function of $\mu=\frac{R}{\left(L_{0} l^{\delta}\right)^{\frac{1}{1+\delta}}}$ in the presence of one fractal extra compactified dimension for $\delta=0.6$. 


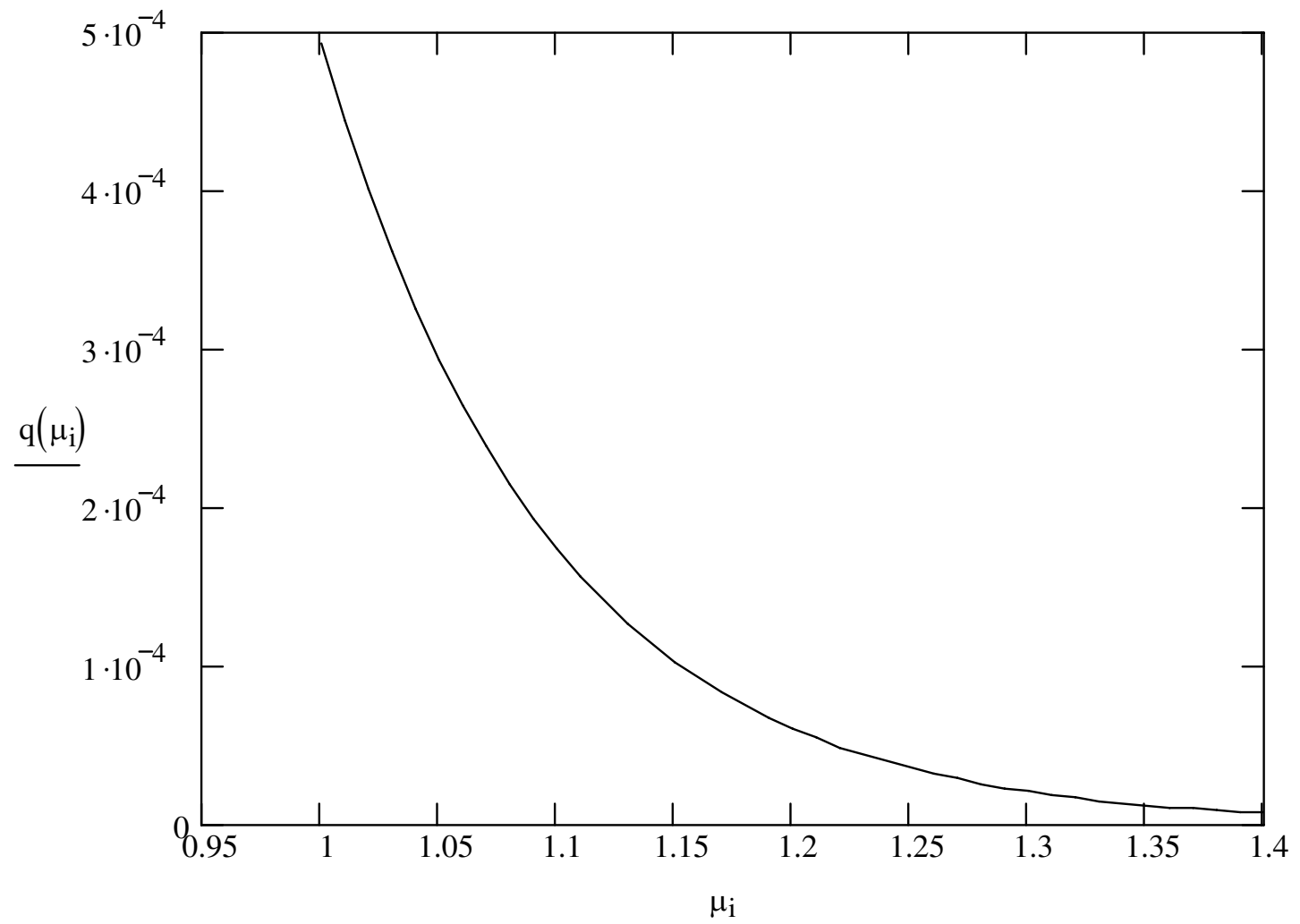

Figure 2: The behaviour of the ratio $q(\mu, \delta)=\frac{C(\mu, \delta)}{f_{0}}$ as a function of $\mu=\frac{R}{\left(L_{0} l^{\delta}\right)^{\frac{1}{1+\delta}}}$ in the presence of one fractal extra compactified dimension for $\delta=0.6$. 


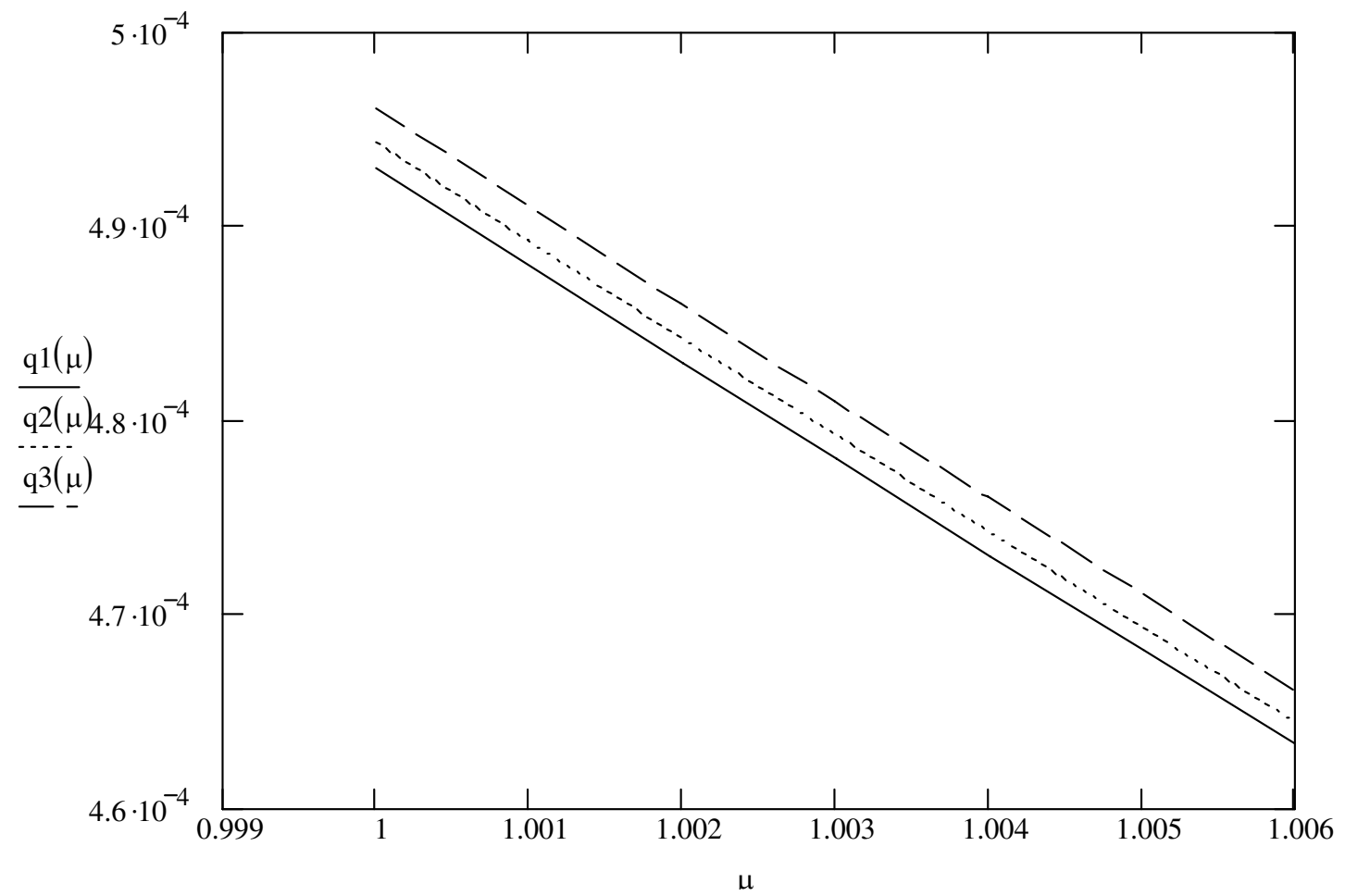

Figure 3: The solid, dot, dashed curves of the ratio $q(\mu, \delta)=\frac{C(\mu, \delta)}{f_{0}}$ as a function of $\mu=$ $\frac{R}{\left(L_{0} l^{\delta}\right)^{\frac{1}{1+\delta}}}$ in the presence of one fractal extra compactified dimension for $\delta=0.6,0.75,0.9$ respectively. 